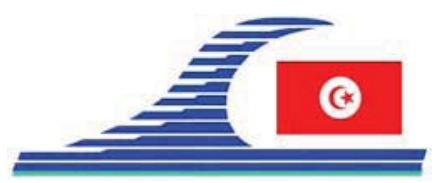

Conférence Méditerranéenne Côtière et Maritime EDITION 1, HAMMAMET, TUNISIE (2009)

Coastal and Maritime Mediterranean Conference

Disponible en ligne - http://www.paralia.fr-Available online

\title{
Prévision de présence et suivi de macro-déchets et méduses en Méditerranée occidentale
}

\author{
Chloé VINCENT ${ }^{1}$, Romain LOUTIER ${ }^{1}$, Olivia LESNE ${ }^{1}$, Antoine MANGIN ${ }^{1}$
}

1. ACRI-ST, 260 route du Pin Montard, BP 234, 06904 Sophia Antipolis Cedex, France.

chloe.vincent@acri-st.fr

\section{Résumé :}

La maîtrise de la qualité des eaux côtières et la gestion des pollutions marines grâce à une meilleure connaissance de leur distribution devient depuis quelques années un enjeu majeur pour les collectivités littorales. L'étude présentée ici consiste à mettre en place des outils de prévision du risque de présence et de dérive de macro-déchets ou de macro-zooplancton -comme les méduses- basés sur des prévisions météorologiques et océaniques opérées en temps quasi-réel. Le principe du modèle de dérive lagrangienne de macro-déchets sera d'abord présenté. Nous nous intéresserons ensuite à la méthode utilisée pour la prévision de présence de méduses à partir des données environnementales et in situ disponibles. Pour la mise en commun des observations de méduses sur le littoral français en Méditerranée, un site internet ouvert à tous a été mis en ligne à l'adresse suivante : http://meduse.acri.fr

\section{Mots-clés :}

Risques Côtiers - GIZC - Environnement littoral - Ecosystèmes côtiers Méditerranée - Méduses - Réseau d'observation - Macro-déchets - Dérive lagrangienne - Rétropropagation

\section{Introduction}

La région Provence-Alpes-Côte-d'Azur (PACA) compte plus de 800 kilomètres de littoral pour une population de 4.7 millions d'habitants. La qualité des eaux de cette région fortement fréquentée est importante pour le tourisme, mais elle est aussi mise en danger par les afflux de population qui en résultent. Nous nous sommes donc attachés au suivi des macro-déchets et des méduses qui peuvent communément peupler ces eaux. L'anticipation de la dérive des macro-déchets et de la présence des méduses permet de mettre en œuvre des outils d'aide à la décision essentiels pour les collectivités côtières.

\section{Modèle de dérive de macro-déchets}

Le modèle de prévision de dérive en mer présenté ici est forcé par les informations météorologiques et océaniques fournies respectivement par les modèles MM5 (DUDHIA et al., 2005) et MARS3D (LAZURE \& DUMAS, 2007) sur la région PACA.

$$
\text { DOI: } 10.5150 / \mathrm{cmcm} .2009 .066-4
$$


MM5 calcule quotidiennement les composantes du vecteur vent à une altitude de $10 \mathrm{~m}$, résolu à $3 \mathrm{~km}$, sur la zone d'intérêt. De même, MARS3D, qui a été mis œuvre en temps quasi-réel dans sa version 6.25 dans le cadre de PREVIMER (http://www.previmer.org), prévoit les courants horizontaux à $400 \mathrm{~m}$ de résolution sur l'ensemble de la colonne d'eau pour la région PACA. Ces deux modèles fournissent des prévisions à $48 \mathrm{~h}$ de l'état du milieu.

Le modèle de dérive de macro-déchets développé est basé sur un algorithme de dérive lagrangienne tridimensionnelle. Il est interfacé avec une application internet dans laquelle l'utilisateur pourra choisir les paramètres clés de la dérive.

Il présente deux modes de fonctionnement : propagation, actuellement en cours de validation, et rétro-propagation, disponible début 2010.

La propagation est la somme d'une vitesse d'advection due à une fraction du courant et une fraction $\mathrm{du}$ vent, d'une contribution aléatoire proportionnelle à la vitesse d'advection matérialisant les effets de dispersion, ainsi que des effets de gravité s'exerçant sur l'objet en mer. Les équations sont discrétisées grâce à un schéma d'Euler explicite. Pour mieux simuler les effets de dispersion, plusieurs particules sont propagées à partir du point d'observation choisi par l'utilisateur pour créer un faisceau de dérives possibles.

Le module de rétro-propagation permettra d'identifier les principales zones de rejet en mer de déchets et de connaitre celles qui conduisent à une dérive ou un échouage systématique dans des périmètres sensibles (réserves naturelles, zones stratégiques, lieux touristiques...). Ainsi les autorités compétentes auront à leur disposition un outil sur lequel s'appuyer pour, par exemple, prendre des mesures en matière d'interdiction de mouillage forain ou de tracé des routes maritimes.

Ce modèle peut également être utilisé pour la dérive de macro-zooplancton: les méduses ne présentent généralement pas de mouvement propre significatif et se laissent porter par l'hydrodynamique du milieu dans lequel elles évoluent. Pourtant, certaines espèces se déplacent verticalement suivant un mouvement nycthéméral. Celui-ci pourra être modélisé par l'ajout d'un terme supplémentaire.

\section{Prévision de présence de méduses en Méditerranée}

En parallèle de l'outil présenté au paragraphe précédent, un modèle de prévision de présence de méduses en Méditerranée est en cours de développement.

A partir des données environnementales caractérisant le milieu et de données in situ de présence de méduses, une étude des corrélations sera mise en place pour connaitre les conditions les plus favorables à la présence de méduse.

Les deux modèles MARS3D et MM5, opérés sur la zone côtière de la région PACA, peuvent fournir les informations nécessaires sur l'état des milieux atmosphériques et océaniques. Cependant, le manque de données d'observation de méduses est limitant. 
C'est pourquoi un réseau d'observation a été créé dans le cadre du projet européen GENESIS (FP7- ICT). Ce réseau est accessible par internet à l'adresse suivante: http://meduse.acri.fr. Il permet à tout utilisateur de communiquer ses observations de présence ou absence de méduse sur l'ensemble des eaux côtières méditerranéennes françaises. Ces informations sont ensuite sauvegardées dans une base de données et une cartographie des méduses observées est proposée aux visiteurs du site internet (Figure 1).

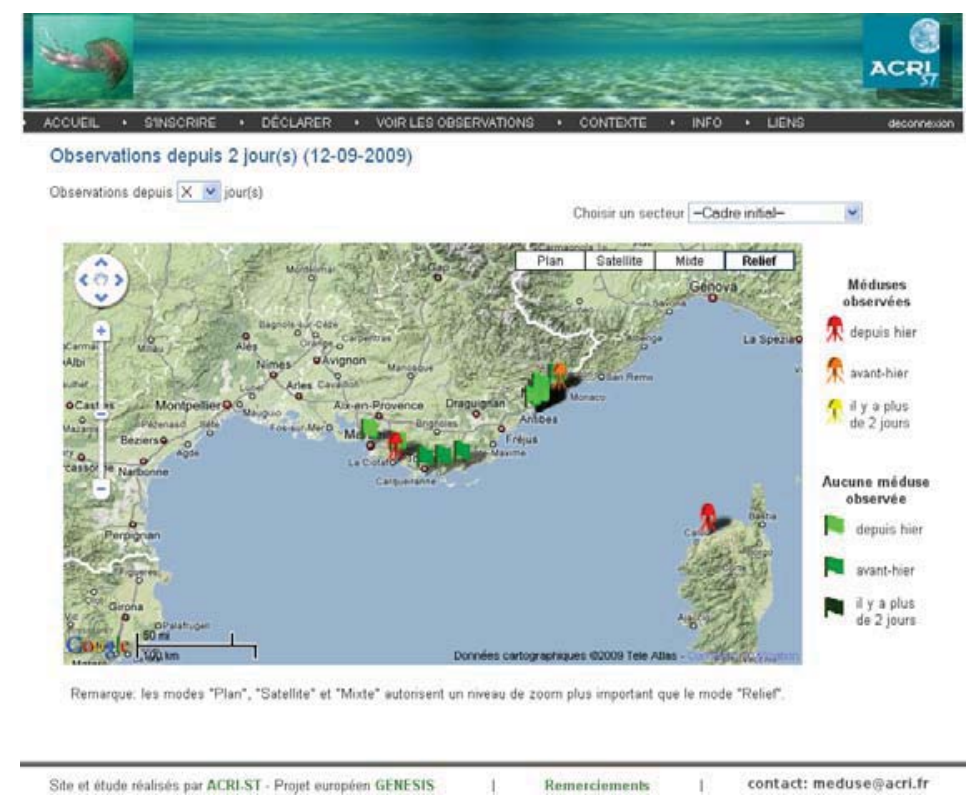

Figure 1. Cartographie des méduses observées les 12 et 13 septembre 2009 source http://meduse.acri.fr

Une probabilité de présence de méduses pourra alors être déduite d'une régression logistique (DECKER et al., 2007), sous forme d'une équation dépendant des paramètres environnementaux dominants issus de l'analyse multicritère.

\section{Conclusion et perspectives}

Les deux outils présentés sont en cours de mise en œuvre pour fournir des informations complémentaires aux gestionnaires d'un milieu côtier. L'étude de corrélation entre environnement et présence de zooplancton aide à mieux connaitre le comportement de ces organismes et à prévoir de possibles apparitions massives. Les paramètres de l'algorithme peuvent être ajustés pour adapter le transport à l'espèce biologique choisie, pourvue d'un mouvement restreint mais présentant éventuellement une migration cyclique propre.

L'outil de prévision de dérive permet quant à lui de suivre une situation potentiellement alarmante de présence massive de zooplancton ou de macro-déchets. Le module de 
rétro-propagation rend possible l'identification des sources de rejet en mer et des zones de production du macro-zooplancton et, grâce à des études statistiques, permet de fournir aux gestionnaires des indicateurs pertinents localisant les zones nécessitant une attention particulière pour prévenir toute situation critique. Là aussi le modèle peut être facilement généralisé à d'autres applications comme par exemple le suivi en mer de bactéries dans un souci de qualité des eaux de baignade.

Ces deux modèles sont actuellement développés indépendamment mais les travaux futurs s'orientent vers le forçage du modèle de dérive par les probabilités de présence, ce qui permettra la mise en place d'un service unique de gestion intégrée de la zone côtière adapté aussi bien au suivi du zooplancton qu'aux macro-déchets ou qu'à d'autres agents pathogènes au comportement connu.

\section{Références bibliographiques}

DECKER M.B., BROWN C.W., HOOD R.R., PURCELL J.E., GROSS T.F., MATANOSKI J.C., BANNON R.O., SETZLER-HAMILTON E.M. (2007). Predicting the distribution of the scyphomedusa Chrysaora quinquecirrha in Chesapeake Bay. Mar. Eco. Prog. Series, Vol. 329, pp 99-113.

DUDHIA J., GILL D., MANNING K., WANG W., BRUYERE C. (2005). PSU/NCAR Mesoscale Modeling System Tutorial Class Notes and Users' Guide (MM5 Modeling System Version 3). Disponible en ligne sur :

[URL : http://www.mmm.ucar.edu/mm5/documents/tutorial-v3-notes.html]

LAZURE P., DUMAS F. (2007). An external-internal mode coupling for a 3D hydrodynamical model for applications at regional scale (MARS). Adv. Water Res., doi:10.1016/j.advwatres.2007.06.010

PREVIMER (site web). http://www.previmer.org 